\title{
Integrin-independent movement of immune cells
}

\section{Sophie E Pinner and Erik Sahai*}

\author{
Address: Cancer Research UK London Research Institute, 44 Lincoln's Inn Fields, London WC2A 3PX, UK \\ *Corresponding author: Erik Sahai (erik.sahai@cancer.org.uk) \\ FI000 Biology Reports 2009, I:67 (doi:10.34I0/BI-67)
}

The electronic version of this article is the complete one and can be found at: http://FI000.com/Reports/Biology/content/I/67

\begin{abstract}
Cell motility requires the temporal and spatial coordination of the actin cytoskeleton with cell-matrix adhesions. Since their discovery more than 20 years ago, integrins have been at the center of cellmatrix adhesion research. Integrin-mediated adhesions link the actin network to the extracellular matrix and are commonly observed as cells migrate across rigid two-dimensional substrates. However, as more cell motility studies are being conducted in three-dimensional (3D) culture systems and in vivo, the role of integrins has become less clear. Recent work has shown that leukocyte migration in 3D contexts can be integrin-independent and that alternative mechanisms of cell adhesion are employed.
\end{abstract}

\section{Introduction and context Integrins}

Integrins are a large family of heterodimeric cell-surface receptors comprising one $\alpha$ and one $\beta$ chain. To bind to ligands, integrins must first undergo a conformation change in a process known as activation [1]. In leukocytes, these conformational changes can be initiated by signaling downstream of other agonists such as chemokines [2]. Activated integrins cluster on the plasma membrane into small focal complexes or larger focal adhesions that are associated with the actin cytoskeleton $[3,4]$. Integrin-based adhesion complexes can bind to either extracellular matrix proteins or proteins present on the surface of other cells. By directly connecting the cytoskeleton to surrounding matrix, integrin-mediated adhesions permit contractile forces to be directly applied to the adhesion to drive locomotion [5]. Integrin complexes are capable of adhering strongly to substrates; for example, the interaction between integrin $\alpha_{L} \beta_{2}$ (lymphocyte function-associated antigen-1, or LFA-1) and intercellular adhesion molecule-1 (ICAM-1) on endothelial cells is strong enough for leukocytes to stick to endothelial cells in the presence of shear forces [6,7]. The relationship between adhesion and cell motility is complex: many studies have shown that an intermediate amount of adhesion is optimal for cell migration; too much adhesion and cells cannot detach to move [8]. Conversely, if there is too little adhesion then cells cannot generate sufficient traction for locomotion [8].

\section{The role of leukocyte motility in immune responses}

The recruitment of leukocytes from the circulation is critical for an effective immune response. First, leukocytes such as dendritic cells (DCs) need to move through peripheral tissues to 'scan' for signs of infection. After antigen uptake, they leave the tissue, enter lymphatic vessels, and travel large distances to reach lymph nodes [9]. Inside lymph nodes, cell motility continues in order for the antigen-presenting DC to find and activate the naïve $\mathrm{T}$ lymphocytes that recognise its unique major histocompatibility complex (MHC)-peptide complex. This process requires the scanning of thousands of lymphocytes, which are also migrating in search of the appropriate DC [10]. The activation of the lymphocyte promotes its sustained proliferation, and the expanding cohort of $\mathrm{T}$ lymphocytes leaves the lymph node and travels to the site of infection to eliminate the potential threat. The transit of these cells and other leukocytes to sites of inflammation is largely through the blood. Endothelial cells in inflamed tissues upregulate chemokines and adhesion molecules such as selectins in 
order to recruit leukocytes [11]. Selectins mediate intermediate-strength contacts with leukocytes that lead to leukocytes rolling along the endothelium [12]. Subsequently, integrins become activated, leading to the formation of firm adhesions that cause leukocyte arrest [12]. The critical role for integrins in this process is demonstrated by the immunodeficiency of patients with defective integrin $\alpha_{\mathrm{L}} \beta_{2}$ (LFA-1) and Kindlin-3 [13]. Thus, immune responses require cell motility to detect infection and for different cell types to meet and relay messages and, finally, to respond to the threat [14].

For an effective immune response, cell motility must be quick and efficient. Leukocytes move at speeds of greater than $10 \mu \mathrm{m} /$ minute, which are an order of magnitude greater than the speed of cell types such as fibroblasts and epithelial cells [15]. These differing speeds of migration are also reflected in the differing organisation of integrin-mediated adhesions on two-dimensional (2D) substrates. Fibroblasts have large integrin-rich focal adhesions that turn over in a time frame of 10-30 minutes $[3,16]$. The longevity of focal adhesions would be incompatible with the high migration speeds of leukocytes, and focal adhesions are not observed in leukocytes. Instead, very transient integrin complexes are present at the front of migrating leukocytes and a larger focal zone of integrins is observed in the middle of the cell [17]. Another challenge facing leukocytes is that they migrate through varying tissues containing different types of extracellular matrix. This problem could be overcome by using integrins that can bind to a broad range of ligands. Consistent with this notion, the leukocyte integrin beta 2 can bind to numerous different ligands, including plasma proteins (Factor X, C3bi, fibrinogen, and fibronectin) [18], extracellular matrix proteins (collagen and laminin) [19,20], and other carbohydrate structures (including heparin-like glycosaminoglycans) [21]. Alternatively, adhesion molecules besides integrins could be used. So what exactly is the role of integrins in leukocyte migration?

\section{Major recent advances}

\section{Integrins are dispensable in certain contexts}

The role of integrins in the interstitial migration of leukocytes was recently investigated by Lämmermann and colleagues [22], who genetically deleted all known integrin heterodimers and found that DCs were still able to migrate from tissue to lymph nodes unimpeded. Using two-photon microscopy, the authors confirmed that the motile behaviour of DCs in the skin and lymph nodes was indistinguishable from that of wild-type DCs [22]. Furthermore, entry of DCs into lymphatic vessels and movement through reconstituted three-dimensional (3D) matrices were unchanged in the absence of integrins [22]. These findings were not specific to DCs as integrins were dispensable for the movement of neutrophils and $\mathrm{B}$ cells in certain contexts. It has also been reported that $\mathrm{T}$ cells can move in the presence of integrin function-blocking antibodies [23]. However, extravasation of integrin-null DCs from the bloodstream to sites of inflammation was completely abolished, underlining the critical role for integrins in immune responses. The critical role of integrins in exit from the blood probably reflects the need for strong adhesions to counteract the shear stresses caused by blood flow. As expected, integrin depletion severely compromised the ability of leukocytes to adhere to ICAM-1 and fibronectin and move in 2D environments. On first inspection, these data suggest that the bi-phasic relationship of adhesion strength and motility may not apply in 3D contexts [24]. However, it is worth noting that the assays used to measure cell adhesion use planar 2D substrates that may not accurately reflect adhesion in $3 \mathrm{D}$ environments.

The mechanism described for this integrin-independent motility of DCs was critically dependent on actin polymerisation. ROCK (Rho kinase)- and myosin II-driven contraction of the actomyosin network was needed to couple the zone of actin polymerisation at the front of the cell to the nucleus and the rest of the cell body [22]. Forces applied by the actomyosin cytoskeleton could also deform the nucleus to allow squeezing through small spaces. This type of motility is similar to that of amoeboid cancer cells [25]. Further work from the same research group has shown a vital role of the small Rho GTPase Cdc42 in the coordination of protrusive activity required for efficient directional migration [26]. Undoubtedly, the mechanisms of leukocyte migration through the interstitium in vivo are just the beginning to be uncovered.

If integrins are dispensable for DC migration in some $3 \mathrm{D}$ environments, then how do the cells adhere to their surroundings? An important observation by Lämmermann and colleagues was that migration on a $2 \mathrm{D}$ plane requires integrins whereas migration between two 2D planes does not. Therefore, a confined environment is all that is needed to permit integrin-independent motility. An exact explanation for this phenomenon is not provided. One possibility is that, if a moving cell is confined around its lateral edges, it can push outwards and increase the friction provided by relatively weak non-integrin interactions between the cell surface and the confining substrates. The increased friction is then sufficient for traction. Conceptually, this is similar to the 'chimneying' technique used by climbers (Figure 1). In the absence of confinement, the cell is unable to effectively push against its substrate. The lateral 
Figure I. How weak adhesion might enable traction in confined spaces but not in open spaces
a)
b)
C)
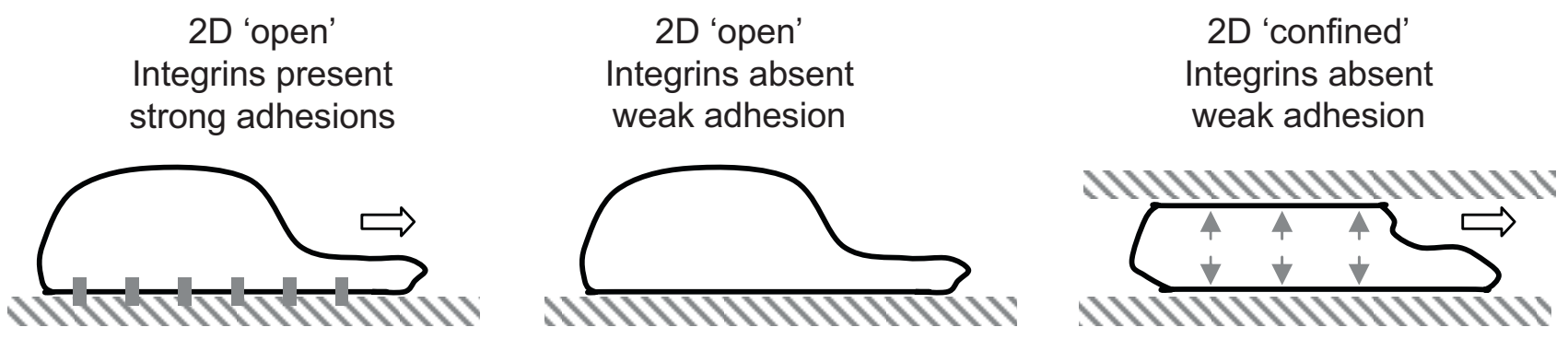

man climbing a ladder
(good grip)

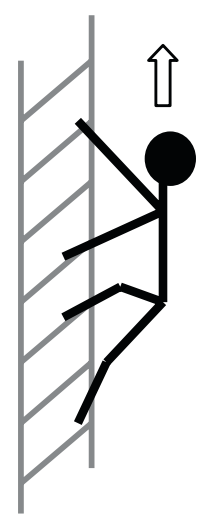

man trying to climb wall (poor grip)

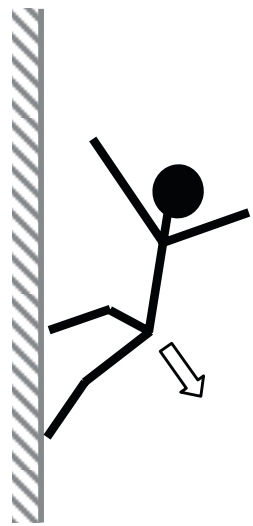

man trying to climb between two walls

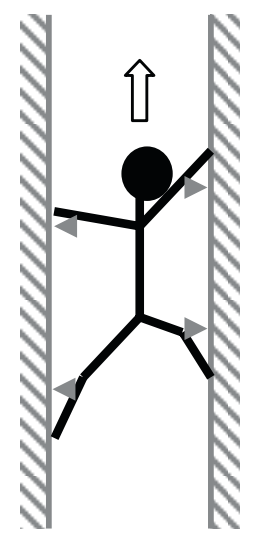

(a) Strong adhesions enable actin polymerisation to drive the front of the cell forward (analogous to a man climbing a ladder). The main limitation is remodelling adhesions to enable the rear to follow. (b) If very weak adhesions are present, then there is insufficient friction and actin polymerisation cannot push the cell forward (analogous to a man trying to climb a smooth wall). (c) Friction generated from weak adhesions can be increased if the cells are pushed against substrate. This can happen in a confined environment (analogous to man climbing between two walls, or 'chimneying').

extension of protrusions into gaps in the matrix may also provide some traction [27].

While the study of Lämmermann and colleagues demonstrates that integrins are not needed for motility in certain contexts, it does not demonstrate that wildtype cells move without using their integrins or provide alternative adhesion molecules. Insights into these issues were provided by Woolf and colleagues [28] in a study of T-cell migration within lymph nodes. The stromal cells of the lymph node such as fibroblastic reticular cells (FRCs) and the endothelium of the high endothelial venules (HEVs) can present both immobilised chemokines, such as chemokine (C-C motif) ligand-21 (CCL21), and integrin ligands such as VCAM-1 (vascular cell adhesion molecule-1) and ICAM-1. However, engagement of integrins within the lymph node could lead to excess adhesion and T-cell arrest before they encountered their specific antigen-presenting cell (APC), resulting in an impaired immune response. To dissect this issue, the roles of immobilised chemokines and integrin ligands were investigated in vitro. It was found that only immobilised chemokines were able to promote sustained T-cell motility and that this did not require additional adhesive signals such as integrin $\beta 1$ engagement [28]. Immobilised CCL21 could induce clustering of integrin $\alpha_{4} \beta_{1}$ (very late antigen-4, or VLA-4) and integrin $\alpha_{\mathrm{L}} \beta_{2}$ (LFA-1) on T cells; critically, however, although these integrins were clustered, they were not adhesive [28]. Furthermore, integrin-blocking antibodies 
could not prevent $\mathrm{T}$ lymphocytes moving within lymph nodes. Therefore, in these contexts, wild-type cells appear to move without using integrins, hence deletion of integrins does not affect motility within lymph nodes [22]. It is possible that the interaction between immobilised CCL21 and its binding partners on the cell surface, CCR7 [chemokine (C-C motif) receptor-7] and PSGL-1 (P-selectin glycoprotein ligand-1), could provide the adhesion necessary for cell migration, although this has not been demonstrated. Adhesion through integrins is induced only when the cells encounter shear forces in addition to chemokine signaling, such as $\mathrm{T}$ cells would encounter on the surface of HEVs when entering the lymph node from the circulation. The lack of integrin adhesiveness in the absence of shear flow has been termed 'integrin silencing' and provides an answer to the apparent contradiction of the presence of both integrin ligands and chemokines on FRCs where T cells need to be highly motile until they find their APC partner. Integrin silencing also occurs in neutrophils moving on fibrinogen in the absence of shear stress [28], suggesting that it may be a general mechanism in leukocytes. Integrin silencing can be over-ridden by strong signaling through the T-cell receptor [28]. Thus, once the T cell is presented with its cognate antigen, strong adhesions stop it from moving.

\section{Future directions}

Before these studies, it had been assumed that integrins were critical for leukocyte motility in all contexts. However, we now have a clearer framework of when integrins are used and when they are not. In situations in which cells need adhesions strong enough to halt motile behaviour and anchor cells in one place, integrins are critical. However, integrins are dispensable for movement through tissues at high speeds and to 'scan' large numbers of potential partners in a lymph node. Although integrins seem to be redundant in these contexts, it does not mean that migrating leukocytes do not require any form of adhesion. For actin polymerisation-driven changes in cell morphology to be successfully translated into locomotion, leukocytes may employ alternative adhesion molecules with lower affinities for their substrates. It is possible that chemokine-receptor interactions may fulfill this role but this is unproven as yet. These weaker adhesions would favour more transient interactions with matrix proteins and rapid cell motility. Integrins recruit a range of proteins to their cytoplasmic tails that couple to F-actin and these proteins enable the force generated by the actin cytoskeleton to act on the extracellular matrix. It remains to be determined how the actin cytoskeleton might connect to non-integrin adhesions used by rapidly migrating leukocytes. An effective immune response requires leukocytes to use both types of motility and to switch between them at the appropriate times. At present, our insights into the molecular details of how cells switch from integrin-dependent to -independent modes of motility are limited. No doubt, the next few years will yield answers to some of these issues and generate new, unexpected and exciting questions.

\section{Abbreviations}

2D, two-dimensional; 3D, three-dimensional; APC, antigen-presenting cell; CCL21, chemokine (C-C motif) ligand-21; CCR7, chemokine (C-C motif) receptor-7; DC, dendritic cell; FRC, fibroblastic reticular cell; HEV, high endothelial venule; ICAM-1, intercellular adhesion molecule-1; LFA-1, lymphocyte function-associated antigen-1; MHC, major histocompatibility complex; PSGL-1, P-selectin glycoprotein ligand-1; ROCK, Rho kinase; VCAM-1, vascular cell adhesion molecule-1; VLA-4, very late antigen- 4 .

\section{Competing interests}

The authors declare that they have no competing interests.

\section{Acknowledgements}

The authors thank Michael Sixt and Chris Madsen for constructive comments. SEP and ES are funded by Cancer Research UK.

\section{References}

I. Fernandez C, Clark K, Burrows L, Schofield NR, Humphries MJ: Regulation of the extracellular ligand binding activity of integrins. Front Biosci 1998, 3:d684-700.

2. Chigaev A, Waller A, Zwartz GJ, Buranda T, Sklar LA: Regulation of cell adhesion by affinity and conformational unbending of alpha4betal integrin. J Immunol 2007, I 78:6828-39.

3. Webb DJ, Brown CM, Horwitz AF: Illuminating adhesion complexes in migrating cells: moving toward a bright future. Curr Opin Cell Biol 2003, I5:6I4-20.

4. Brakebusch C, Fassler R: The integrin-actin connection, an eternal love affair. EMBO J 2003, 22:2324-33.

5. Friedland JC, Lee $\mathrm{MH}$, Boettiger D: Mechanically activated integrin switch controls alpha5betal function. Science 2009, 323:642-4.

6. Salas A, Shimaoka M, Chen S, Carman CV, Springer T: Transition from rolling to firm adhesion is regulated by the conformation of the I domain of the integrin lymphocyte functionassociated antigen-I. J Biol Chem 2002, 277:50255-62.

7. Salas A, Shimaoka M, Phan U, Kim M, Springer TA: Transition from rolling to firm adhesion can be mimicked by extension of integrin alphaLbeta2 in an intermediate affinity state. J Biol Chem 2006, 281:10876-82.

8. Schwartz MA, Horwitz AR: Integrating adhesion, protrusion, and contraction during cell migration. Cell 2006, I 25: I223-5.

9. Randolph GJ, Angeli V, Swartz MA: Dendritic-cell trafficking to lymph nodes through lymphatic vessels. Nat Rev Immunol 2005, 5:617-28

10. Miller MJ, Hejazi AS, Wei SH, Cahalan MD, Parker I: T cell repertoire scanning is promoted by dynamic dendritic cell 
behavior and random $\mathrm{T}$ cell motility in the lymph node. Proc Natl Acad Sci U S A 2004, 101:998-1003.

FI000 Factor 4.9 Must Read

Evaluated by Michael Dustin 03 Feb 2004, Rick Tarleton 24 Feb 2004, Andrea Sant 10 Aug 2004

II. Johnston B, Butcher EC: Chemokines in rapid leukocyte adhesion triggering and migration. Semin Immunol 2002, 14:83-92.

12. Alon $\mathrm{R}$, Ley $\mathrm{K}$ : Cells on the run: shear-regulated integrin activation in leukocyte rolling and arrest on endothelial cells. Curr Opin Cell Biol 2008, 20:525-32.

13. Svensson L, Howarth K, McDowall A, Patzak I, Evans R, Ussar S, Moser M, Metin A, Fried M, Tomlinson I, Hogg N: Leukocyte adhesion deficiency-III is caused by mutations in KINDLIN3 affecting integrin activation. Nat Med 2009, 15:306-I2.

FI000 Factor 3.0 Recommended

Evaluated by Thomas Egelhoff 12 May 2009

14. Teoh D, Johnson LA, Hanke T, McMichael AJ, Jackson DG: Blocking development of a $\mathrm{CD8}^{+} \mathrm{T}$ cell response by targeting lymphatic recruitment of APC. J Immunol 2009, I82:2425-3I.

15. Kolega J, Shure MS, Chen WT, Young ND: Rapid cellular translocation is related to close contacts formed between various cultured cells and their substrata. I Cell Sci 1982, 54:23-34.

16. Holt MR, Calle Y, Sutton DH, Critchley DR, Jones GE, Dunn GA: Quantifying cell-matrix adhesion dynamics in living cells using interference reflection microscopy. J Microsc 2008, 232:73-8I.

17. Smith A, Carrasco YR, Stanley P, Kieffer N, Batista FD, Hogg N: A talin-dependent LFA-I focal zone is formed by rapidly migrating T lymphocytes. J Cell Biol 2005, I70:|4|-5I.

FI000 Factor 3.2 Recommended

Evaluated by Kenneth Yamada 27 Jul 2005, Eric Brown 16 Aug 2005

18. Sadhu C, Ting HJ, Lipsky B, Hensley K, Garcia-Martinez LF, Simon SI, Staunton DE: CD I I C/CD I 8: novel ligands and a role in delayedtype hypersensitivity. J Leukoc Biol 2007, 81:1395-403.

19. Penberthy TW, Jiang Y, Luscinskas FW, Graves DT: MCP-Istimulated monocytes preferentially utilize beta 2-integrins to migrate on laminin and fibronectin. Am J Physiol 1995, 269: C60-8.

20. Walzog B, Schuppan D, Heimpel C, Hafezi-Moghadam A, Gaehtgens P, Ley $\mathrm{K}$ : The leukocyte integrin Mac-I (CDIIb/CDI8) contributes to binding of human granulocytes to collagen. Exp Cell Res 1995, 218:28-38.
2I. Vorup-Jensen T, Chi L, Gjelstrup LC, Jensen UB, Jewett CA, Xie C, Shimaoka M, Linhardt RJ, Springer TA: Binding between the integrin alphaXbeta2 (CDI I C/CD I8) and heparin. J Biol Chem 2007, 282:30869-77.

22. Lämmermann T, Bader BL, Monkley SJ, Worbs T, Wedlich-Söldner R, Hirsch K, Keller M, Förster R, Critchley DR, Fässler R, Sixt M: Rapid leukocyte migration by integrin-independent flowing and squeezing. Nature 2008, 453:5I-5.

FI000 Factor 10.8 Exceptional

Evaluated by Friedemann Kiefer 06 May 2008, Kenneth Yamada 09 May 2008, Jens V Stein 13 May 2008, Vivian Tang 23 May 2008, Carol Otey 09 Jul 2008, Jia-huai Wang 09 Jul 2008, Thomas Egelhoff 16 Sep 2008

23. Friedl P, Entschladen F, Conrad C, Niggemann B, Zanker KS: CD4 ${ }^{+} \mathbf{T}$ lymphocytes migrating in three-dimensional collagen lattices lack focal adhesions and utilize betal integrin-independent strategies for polarization, interaction with collagen fibers and locomotion. Eur J Immunol 1998, 28:233I-43.

24. Doyle AD, Wang FW, Matsumoto K, Yamada KM: One-dimensional topography underlies three-dimensional fibrillar cell migration. J Cell Biol 2009, I84:48I-90.

FI000 Factor 5.0 Must Read

Evaluated by Thomas Egelhoff 27 Feb 2009, Charles Streuli 09 Mar 2009, Martin Humphries 27 Mar 2009, Martin A Schwartz 08 Apr 2009

25. Wyckoff JB, Pinner SE, Gschmeissner S, Condeelis JS, Sahai E: ROCKand myosin-dependent matrix deformation enables protease-independent tumor-cell invasion in vivo. Curr Biol 2006, 16:1515-23.

FI000 Factor 3.0 Recommended Evaluated by Peter Friedl 29 Sep 2006

26. Lämmermann T, Renkawitz J, Wu X, Hirsch K, Brakebusch C, Sixt M: Cdc42-dependent leading edge coordination is essential for interstitial dendritic cell migration. Blood 2009, I I 3:5703-10.

27. Mandeville JT, Lawson MA, Maxfield FR: Dynamic imaging of neutrophil migration in three dimensions: mechanical interactions between cells and matrix. J Leukoc Biol 1997, 6I:188-200.

28. Woolf E, Grigorova I, Sagiv A, Grabovsky V, Feigelson SW, Shulman Z, Hartmann T, Sixt M, Cyster JG, Alon R: Lymph node chemokines promote sustained $T$ lymphocyte motility without triggering stable integrin adhesiveness in the absence of shear forces. Nat Immunol 2007, 8:1076-85.

FI000 Factor 6.0 Must Read

Evaluated by Michael Dustin 03 Oct 2007 